\title{
CARACTERIZAÇÃO DAS VARIÁVEIS HIDROQUÍMICAS NA SUB-BACIA DO ALTO JAGUARIBE, CEARÁ UTILIZANDO ANÁLISE MULTIVARIADA E SIG
}

\author{
Eldir Bandeira da Silva ${ }^{1}$, José Ribeiro de Araújo Neto ${ }^{2}$
}

\begin{abstract}
RESUMO
A avaliação da qualidade das águas superficiais e deteç̧ão de possíveis degradações é de fundamental importância para monitoramento do manejo de bacias hidrográficas. Com isso objetivou-se com este trabalho avaliar a qualidade das águas superficiais dos reservatórios da bacia do Alto Jaguaribe, Ceará, quanto aos parâmetro químicos para fins de irrigação. O estudo foi realizado em 10 reservatórios que fazem parte sub-bacia do Alto Jaguaribe. Foram utilizados os dados das concentrações químicas das águas dos reservatórios em um período de 2001 a 2015 dos seguintes parâmetros: condutividade elétrica $(\mathrm{CE})$, cloreto $(\mathrm{Cl})$, sódio $\left(\mathrm{Na}^{+}\right)$, cálcio $\left(\mathrm{Ca}^{2+}\right)$ e magnésio $\left(\mathrm{Mg}^{2+}\right)$, totalizando 750 amostras. Os dados foram provenientes do banco de dados da Companhia de Gestão dos Recursos Hídricos (COGERH) disponibilizados ao Instituto Federal de Educação, Ciência e Tecnologia do estado do Ceará, campus Iguatu. Foi aplicado a técnica de Estatística Multivariada Análise de Agrupamento Hierárquico (AAH) para formação da similaridade entre os reservatórios. De acordo com resultados, as águas dos reservatórios da sub-bacia do Alto Jaguaribe apresentam até o momento, moderado risco à salinidade de maneira geral, com exceção dos reservatórios da região do Inhamuns, em que foram encontrados elevadas concentrações de sais. Os açudes Broco e Favelas obtiveram as maiores concentrações de sais dentre os estudados, com valores acima dos limites para consumo humano e irrigação, fato ocorrido pela baixa capacidade dos reservatórios, elevada taxa de evaporação e baixa pluviosidade, acarretando aumento da concentração de sais do corpo hídrico.
\end{abstract}

Palavras-chave: semiárido, salinidade, geoprocessamento, estatística.

\section{ABSTRACT \\ SPATIALIZATION OF WATER QUALITY OF SURFACE HIGH JAGUARIBE BASIN, CEARÁ FOR PURPOSES OF IRRIGATION}

Evaluation of surface water quality and detection of possible degradations of fundamental importance for the monitoring of river basin management. Thereby the objective of this work was to evaluate the quality of surface water reservoirs in the basin of the Alto Jaguaribe, Ceará as the chemical parameter for irrigation purposes. The study was conducted in 10 reservoirs that are part of sub-basin of the Alto Jaguaribe. the data of chemical concentrations of the reservoir water were used in a period from 2001 to 2015 the following parameters: electrical conductivity (EC), chloride ( $\mathrm{Cl}^{-}$ ), sodium $\left(\mathrm{Na}^{+}\right)$, calcium $\left(\mathrm{Ca}^{2+}\right)$ and magnesium $\left(\mathrm{Mg}^{2+}\right)$, totaling 750 samples. The data came from the company's database Water Management (COGERH) available to the Federal Institute of Education, Science and Technology of Ceará state, campus Iguatu. The statistical technique was applied Multivariate Hierarchical Cluster Analysis (HCA) for formation of the similarity between the reservoirs. According to results, the waters of the Alto Jaguaribe sub-basin reservoirs have so far, moderate risk to salinity in general, with the exception of shells of Inhamuns region, in which were found high concentrations of salts. The Broco and Favelas weirs obtained the highest concentrations of salts from the study, with the upper limit for human consumption and irrigation, fact occurred by the low capacity of the reservoirs, high evaporation and low rainfall, resulting in increased concentration of body salts water.

Keywords: semiarid, salinity, geoprocessing, statistic.

\section{Recebido para publicação em 05/06/2016. Aprovado em 19/10/2016.}

1 - Tecnólogo em Irrigação e Drenagem, Mestrando, UFC/Fortaleza, Departamento de Eng. Agrícola, eldir_2005@hotmail.com 2 - Tecnólogo em Irrigação e Drenagem, Técnico do Laboratório de Solo, Água e Tecido Vegetal - IFCE/Iguatu, Doutorando, UFC/ Fortaleza, Departamento de Engenharia Agrícola, juniorifcelabas@gmail.com 


\section{INTRODUÇ̃̃O}

O semiárido brasileiro é caracterizado pela distribuição irregular da precipitação pluviométrica no tempo e no espaço e elevadas taxas evapotranspiratórias, levando à prática da construção de reservatórios de captação e armazenamento das águas superficiais para abastecimento em geral (FERREIRA et al., 2015). $\mathrm{O}$ crescimento populacional e o desenvolvimento socioeconômico ocasionam o aumento da demanda hídrica (BUENO et al., 2005).

As águas armazenadas em reservatórios superficiais são as principais fontes para múltiplos usos como, demandas por consumo humano, animal, indústrias e, sobretudo, da agricultura irrigada. No entanto, essas águas são provenientes de escoamentos superficiais e sub superficiais oriundas das redes de drenagem, resultante da precipitação sobre uma área, que acarreta no arrastre de nutrientes e minerais para o corpo hídrico e, por conseguinte, incremento de sais no reservatório (SILVA JÚNIOR et al., 2000). Um dos problemas ocasionados pelo arraste de sais para os reservatórios é a salinização (ARAÚJO NETO et al., 2014).

Os sais presentes nas águas têm sua origem na intemperização das rochas e dos solos, que são levados pelas águas de irrigação e se depositam no solo, acumulando à medida que a água evapora (AYERS; WESTCOT, 1999). Outra fonte de acumulação de sais é a ação antrópica sobre os corpos hídricos (FROTA JUNIOR et al., 2007). Para Barbosa (2006), são as ações antrópicas que vêm causando grandes transformações no meio natural, atingindo severamente o ecossistema. Esse aumento nos valores de sais nas água é diagnosticado, principalmente, pela elevação nos

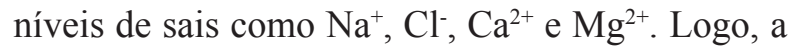
determinação da qualidade da água é fundamental para dimensionamento de projetos agronômicos (ARRAES et al., 2009).

Diversas medições de variáveis são requeridas para avaliação da qualidade de água de determinado reservatório, e para haver a relação e similaridade entre as variáveis a técnica de estatística multivariada análise de agrupamento vem sendo empregado por diversos pesquisadores (FECHINE; GALVÍNCIO, 2008; LOPES et al., 2009; PALÁCIO et al., 2009) por permitir uma visão mais global dos fenômenos (SALGADO et al., 2011).

Considerando a atual situação dos reservatórios quanto a salinização, objetivou-se com este trabalho caracterizar a qualidade das águas superficiais dos reservatórios da bacia do Alto Jaguaribe, Ceará por intermédio de ferramentas estatísticas e de geoprocessamento para fins agronômicos.

\section{MATERIAL E MÉTODOS}

O estudo foi realizado na sub-bacia do Alto Jaguaribe, Ceará, entre as latitudes $5^{\circ} 54^{\prime} 55^{\prime \prime}$ e $7^{\circ} 04^{\prime} 50^{\prime \prime} \mathrm{S}$ e as longitudes $38^{\circ} 55^{\prime} 22^{\prime \prime}$ e $40^{\circ} 24^{\prime} 47^{\prime \prime}$ W (Quadro 1). Os 10 reservatórios que deram suporte para esta pesquisa foram: Arneiroz II, Bengué, Broco, Do Coronel, Favelas, Forquilhas II, Orós, Pau Preto, Trici e Trussu (Figura 1).

A evapotranspiração potencial da região oscila entre 1.500 e $2.000 \mathrm{~mm}^{2} \mathrm{ano}^{-1} \mathrm{e}$ as temperaturas médias anuais registram valores de 23 a $27{ }^{\circ} \mathrm{C}$. A umidade relativa média é de $50 \%$ e o período de insolação chega a $2.800 \mathrm{~h}$ ano-1 (ARAÚJO NETO et al., 2014). A precipitação pluviométrica na região varia entre 549,6 a 1009,2 $\mathrm{mm}^{-1} \mathrm{ano}^{-1}$ de acordo com a Fundação Cearense de Meteorologia e Recursos Hídricos (FUNCEME) entre os anos de 1974 a 2015 (Figura 2).

Os dados das concentrações químicas das águas dos reservatórios para o período de 2001/2015, num total de 750 amostras utilizados no presente estudo, foram provenientes do banco de dados da Companhia de Gestão dos Recursos Hídricos (COGERH) disponibilizados ao Instituto Federal de Educação, Ciência e Tecnologia do estado do Ceará, campus Iguatu. Os atributos químicos considerados nesse estudo foram os teores de: condutividade elétrica $(\mathrm{CE})$, cloreto $\left(\mathrm{Cl}^{-}\right)$, sódio $\left(\mathrm{Na}^{+}\right)$, cálcio $\left(\mathrm{Ca}^{2+}\right)$ e magnésio $\left(\mathrm{Mg}^{2+}\right)$. Os açudes monitorados na área do estudo foram

\section{REVENG}

$$
\text { 417-426p. }
$$




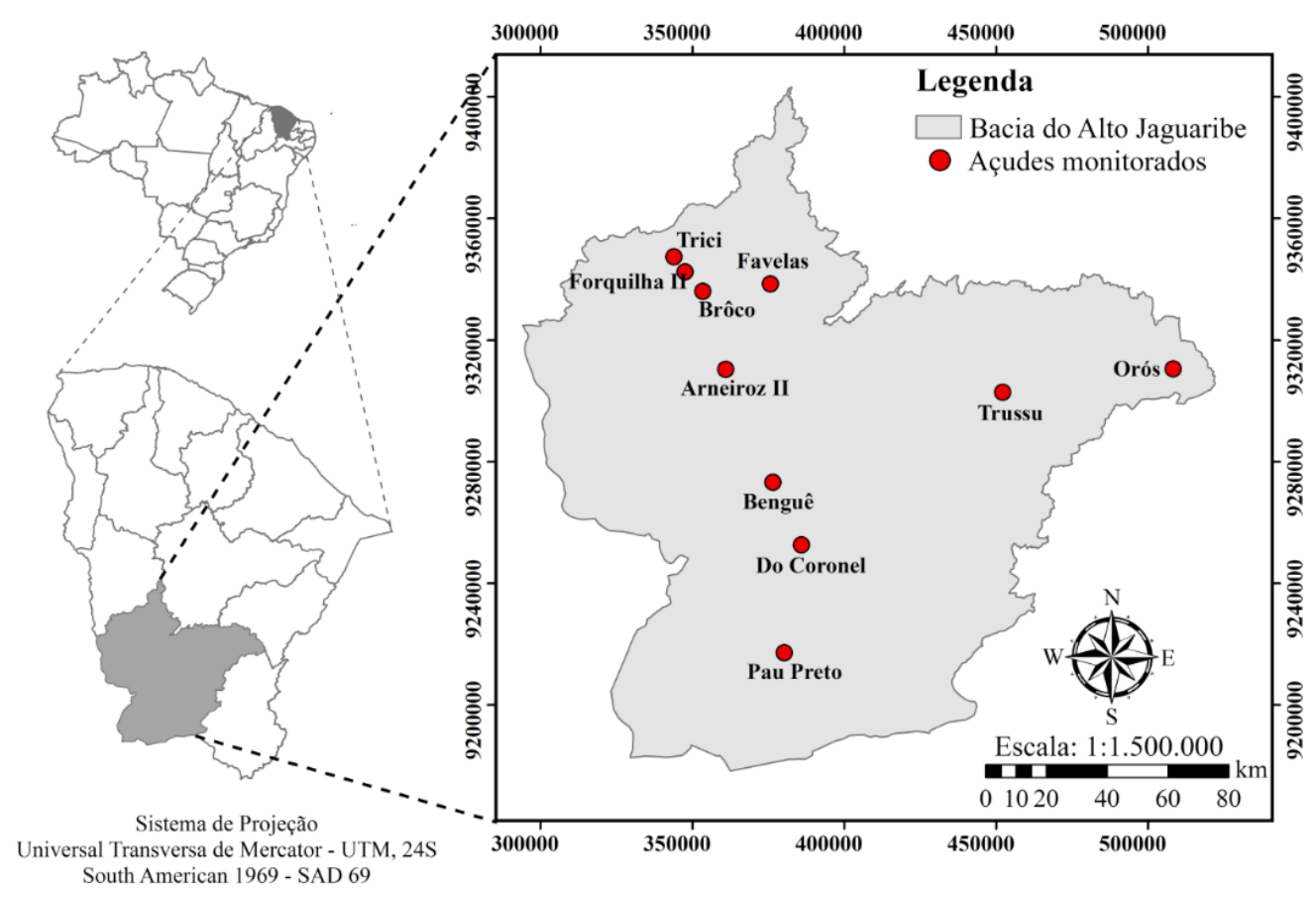

Fonte: Autor.

Figura 1. Localização dos reservatórios na sub-bacia do Alto Jaguaribe, Ceará.

Quadro 1. Características dos reservatórios estudados

\begin{tabular}{|c|c|c|c|c|c|}
\hline \multirow{2}{*}{ Reservatórios } & \multirow{2}{*}{$\begin{array}{l}\text { Capacidade } \\
\left(\mathrm{hm}^{3}\right)\end{array}$} & \multirow{2}{*}{$\begin{array}{l}\text { Precipitação Anual } \\
\text { (mm) }\end{array}$} & \multirow{2}{*}{ Município } & \multicolumn{2}{|c|}{ Localização } \\
\hline & & & & Latitutde & Longitude \\
\hline Arneiroz II & 187,7 & 634,8 & Arneiroz & $6^{\circ} 16^{\prime} 04^{\prime \prime}$ & $40^{\circ} 13^{\prime} 13^{\prime \prime}$ \\
\hline Bengué & 18,0 & 577,2 & Aiuaba & $6^{\circ} 35^{\prime} 46^{\prime \prime}$ & $40^{\circ} 08^{\prime} 22^{\prime \prime}$ \\
\hline Broco & 8,4 & 549,6 & Tauá & $6^{\circ} 00^{\prime} 19^{\prime \prime}$ & $40^{\circ} 19^{\prime} 28^{\prime \prime}$ \\
\hline Do Coronel & 1,7 & 549,6 & Antonina do Norte & $6^{\circ} 38^{\prime} 53^{\prime \prime}$ & $40^{\circ} 03^{\prime} 17^{\prime \prime}$ \\
\hline Favelas & 30,1 & 549,6 & Tauá & $5^{\circ} 57^{\prime} 35^{\prime \prime}$ & $40^{\circ} 12^{\prime} 36^{\prime \prime}$ \\
\hline Forquilha II & 3,4 & 549,6 & Tauá & $5^{\circ} 56^{\prime} 31^{\prime \prime}$ & $40^{\circ} 23^{\prime} 58^{\prime \prime}$ \\
\hline Orós & 1940,0 & 800,4 & Orós & $6^{\circ} 14^{\prime} 10^{\prime \prime}$ & $38^{\circ} 55^{\prime} 22^{\prime \prime}$ \\
\hline Pau Preto & 1,9 & 702,0 & Potengi & $7^{\circ} 04^{\prime} 50^{\prime \prime}$ & $40^{\circ} 05^{\prime} 06^{\prime \prime}$ \\
\hline Trici & 16,5 & 549,6 & Tauá & $5^{\circ} 54^{\prime} 55^{\prime \prime}$ & $40^{\circ} 24^{\prime} 47^{\prime \prime}$ \\
\hline Trussu & 268,8 & 1009,2 & Iguatu & $6^{\circ} 18^{\prime} 32^{\prime \prime}$ & $39^{\circ} 25^{\prime} 47^{\prime \prime}$ \\
\hline
\end{tabular}

Fonte: Autor.

georeferenciados por intermédio de GPS de Navegação.

Foi aplicada a técnica de análise multivariada de agrupamento hierárquico $(\mathrm{AAH})$ para estimar a similaridade entre as coletas nos dois períodos de monitoramento processada no software SPSS
20.0. Como as variáveis classificatórias adotadas neste estudo são variáveis reais e, portanto, são mensuradas em uma escala de intervalo, foi adotada a combinação da distância Euclidiana ao quadrado para a formação da matriz de similaridade e o algoritmo do método de ligação Ward (método 


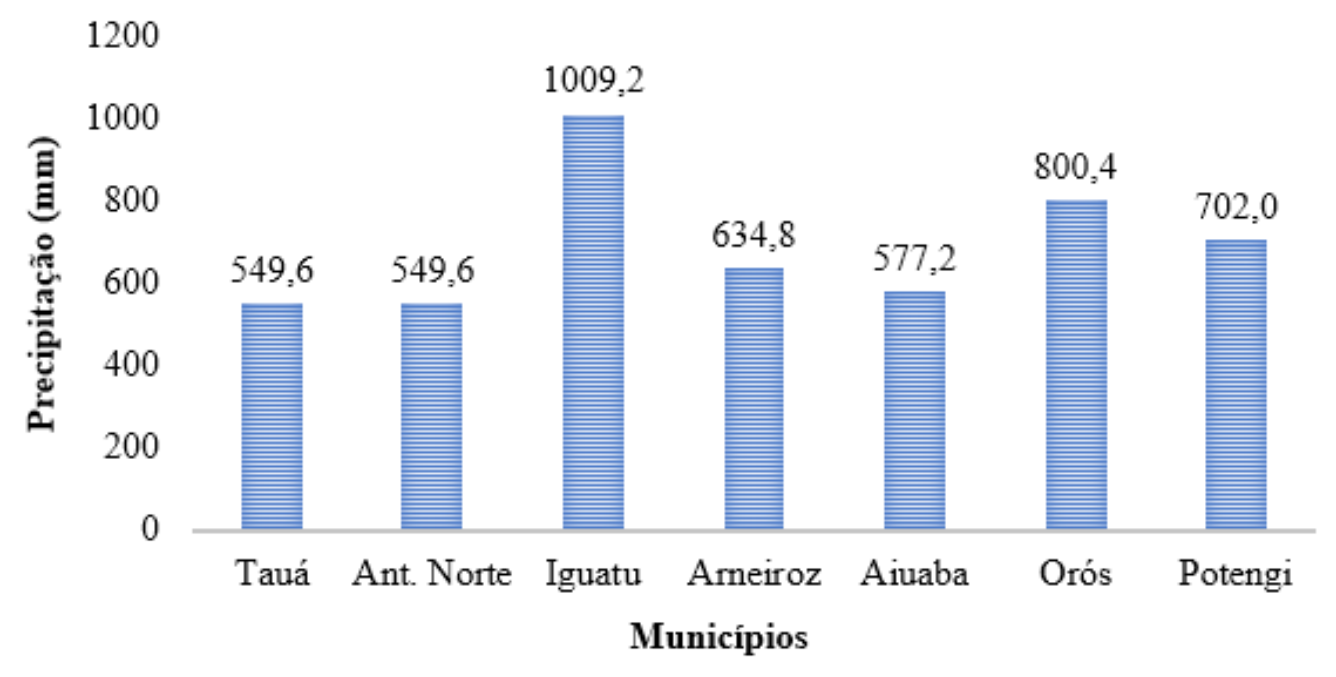

Fonte: Autor.

Figura 2. Média da precipitação das cidades que contemplam os reservatórios entre os anos de 1974 a 2015.

da variância mínima). Para avaliação dos dados médios das variáveis utilizadas para formação dos grupos formados na AAH, foi realizada a análise de variância (ANOVA), com o teste de comparação das médias (teste de Tukey, ao nível de significância de $1 \%$ ) utilizando o programa-SPSS 20.0. for Windows.

Através de técnicas de geoprocessamento e com base nos valores dos parâmetros monitorados, desenvolveram-se as matrizes por meio do método de interpolação por krigagem e variograma linear utilizando o software Surfer v. 8.0. Após obtenção das matrizes de pontos, foram construídos mapas relativos a cada parâmetro no software ArcGIS 9.3.

\section{RESULTADOS E DISCUSSÕES}

No quadro 2, verificou-se a concentração média dos parâmetros estudados para cada reservatório da bacia do Alto Jaguaribe, Ceará. A condutividade elétrica dos reservatórios oscilaram entre 0,268 a $2,158 \mathrm{dS} \mathrm{m}^{-1}$, com valor médio de $0,720 \mathrm{dS} \mathrm{m}^{-1}$. Os resultados de condutividade elétrica restrigem o uso da água tanto para abastecimento humano como para uso na irrigação segundo Ayers e Westcot (1999) $\left(<0,500 \mathrm{dS} \mathrm{m}^{-1}\right.$ para consumo humano e $<0,700 \mathrm{dS} \mathrm{m}^{-1}$ para uso na irrigação). Sales et al. (2014) estudando a salinidade das águas superficiais da bacia metropolitana do estado do Ceará encontraram uma variação semelhante a observada nesse estudo para condutividade elétrica $(0,51$ a $2,15 \mathrm{dS} \mathrm{m}^{-1}$ ). Palácio (2011) relata que as altas taxas de evapotranspiração na região Nordeste do Brasil em conjunto com as baixas alturas pluviométricas corroboram para que haja um acúmulo de sais nos reservatórios. Para Frota Junior (2007), além dos processos naturais climáticos, a ação antrópica é um dos principais causadores do aumento da salinidade nos reservatórios. Concentrações acima

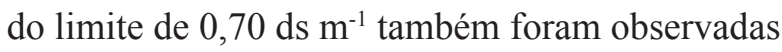
por Mendes et al. (2008) avaliando a qualidade da águas para fins de irrigação da região do Congo, Paraíba.

$\mathrm{O} \mathrm{Na}^{+}$e o $\mathrm{Cl}^{-}$são parâmetros que causam a salinidade e para esse estudo apresentaram valores médios acima do limite estabelecido para irrigação, que é de $3 \mathrm{mmol}_{\mathrm{c}} \mathrm{L}^{-1}$ (AYERS \& WESTCOT, 1999). O íon $\mathrm{Na}^{+}$nos reservatórios apresentou uma variação entre 1,40 a $8,40 \mathrm{mmol}_{\mathrm{c}} \mathrm{L}^{-1}$, e valor médio de 3,23 $\mathrm{mmol}_{\mathrm{c}} \mathrm{L}^{-1}$. Santos et al. (2009) encontraram valores de $\mathrm{Na}^{+}$semelhantes aos encontrados neste estudo avaliando águas superficiais da bacia do Acaraú, Ceará. Em geral, as águas do cristalino do Nordeste do Brasil se classificam como cloretadas sódicas, de acordo com a litologia local (SILVA JUNIOR et al., 2000). Já para o íon $\mathrm{Cl}^{-} \mathrm{o}$ valor médio foi de $3,90 \mathrm{mmol}_{\mathrm{c}} \mathrm{L}^{-1}$, com variação de 0,94

\section{REVENG 417-426p.}


Quadro 2. Valores médios das concentrações dos reservatório da sub-bacia do Alto Jaguaribe, Ceará

\begin{tabular}{lcccccc}
\hline \multirow{2}{*}{ Reservatórios } & \multicolumn{6}{c}{ Valores médios de cada reservatório } \\
\cline { 2 - 7 } & $\begin{array}{c}\mathrm{CE} \\
(\mathrm{dS} / \mathrm{m})\end{array}$ & $\begin{array}{c}\mathrm{Na}^{+} \\
\left(\mathrm{mmol}_{\mathrm{c}} \mathrm{L}^{-1}\right)\end{array}$ & $\begin{array}{c}\mathrm{Cl}^{-} \\
\left(\mathrm{mmol}_{\mathrm{c}} \mathrm{L}^{-1}\right)\end{array}$ & $\begin{array}{c}\mathrm{Ca}^{2+} \\
\left(\mathrm{mmol}_{\mathrm{c}} \mathrm{L}^{-1}\right)\end{array}$ & $\begin{array}{c}\mathrm{Mg}^{2+} \\
\left(\mathrm{mmol}_{\mathrm{c}} \mathrm{L}^{-1}\right)\end{array}$ & $\begin{array}{c}\mathrm{Ca}^{2+}+\mathrm{Mg}^{2+} \\
\left(\mathrm{mmol}_{\mathrm{c}} \mathrm{L}^{-1}\right)\end{array}$ \\
\hline Arneiroz II & 0,519 & 2,84 & 1,96 & 1,44 & 1,39 & 2,84 \\
Bengué & 0,268 & 1,47 & 0,94 & 1,59 & 0,90 & 2,49 \\
Broco & 1,583 & 8,40 & 7,09 & 1,35 & 3,40 & 4,75 \\
Coronel & 0,329 & 1,90 & 1,48 & 1,89 & 0,97 & 2,86 \\
Favelas & 2,158 & 7,08 & 15,94 & 1,30 & 4,72 & 6,02 \\
Forquila II & 0,890 & 2,83 & 5,09 & 2,40 & 0,79 & 3,18 \\
Orós & 0,298 & 1,73 & 1,08 & 1,54 & 1,00 & 2,54 \\
Pau Preto & 0,502 & 2,82 & 2,87 & 2,36 & 1,20 & 3,55 \\
Trici & 0,334 & 1,87 & 1,42 & 1,28 & 1,19 & 2,47 \\
Trussu & 0,321 & 1,40 & 1,13 & 1,60 & 1,06 & 2,66 \\
\hline Mínimo & 0,268 & 1,40 & 0,94 & 1,28 & 0,79 & 2,47 \\
Máximo & 2,158 & 8,40 & 15,94 & 2,40 & 4,72 & 6,02 \\
Médio \pm DP & 0,720 & 3,23 & 3,90 & 1,67 & 1,66 & 3,34 \\
Fonte: Autor. & & & & & &
\end{tabular}

a $15,94 \mathrm{mmol}_{\mathrm{c}} \mathrm{L}^{-1}$. Esse valor médio ultrapassa o limite estabelecido por Ayers e Westcot (1999) (3 mmol $\mathrm{L}^{-1}$ ). Porém Grattan (2005) afirma que culturas sensíveis podem tolerar até $3,4 \mathrm{mmol}_{\mathrm{c}}$ $\mathrm{L}^{-1}$, e culturas mais resistentes até $19,7 \mathrm{mmol}_{\mathrm{c}} \mathrm{L}^{-1}$. Lobato et al. (2008) também encontraram valores de Cloreto superiores ao limite estabelecido, e por isso, causam restrições quanto ao uso na irrigação.

As concentrações dos reservatórios quanto aos parâmetros $\mathrm{Ca}^{2+}$ e $\mathrm{Mg}^{2+}$ variaram entre 1,28 a 2,40 mmol $_{c} \mathrm{~L}^{-1}$ e 0,79 a 4,72 mmol $_{c} \mathrm{~L}^{-1}$, respectivamente. As médias de ambos não apresentaram nenhuma restrição para irrigação segundo os limites estabelecidos por Ayers e Westcot (1999) que é de $5 \mathrm{mmol}_{c} \mathrm{~L}^{-1}$ para Cálcio e $20 \mathrm{mmol}_{\mathrm{c}} \mathrm{L}^{-1}$ para Magnésio. De acordo com os referidos autores, a função do $\mathrm{Ca}^{2+}$ está relacionada a diminuição dos efeitos tóxicos causados pelo $\mathrm{Na}^{+}$e $\mathrm{Mg}^{2+}$ quando absorvidos pelas raízes das plantas, e a função do $\mathrm{Mg}^{2+}$ está relacionada a processos metabólicos ocorridos nas enzimas das plantas. Arraes (2009) alerta que o elemento $\mathrm{Ca}^{2+}$ em altas concentrações nas águas pode causar incrustações nas tubulações de irrigação e, consequentemente, entupimento dos aspersores.
O grupo 1 foi formado pelos reservatórios que possuem as mais baixas concentrações avaliadas, todos localizados na parte central da sub-bacia do Alto Jaguaribe. As concentrações medianas foram as responsáveis pela formação do segundo grupo, composto apenas por dois reservatórios. Já o grupo 3 , composto também por dois reservatórios, foi formado por possuírem as concentrações mais elevadas dentre eles (Figura 3).

No grupo 1, formado pelos reservatórios Bengué, Trussu, Orós, Coronel, Arneiroz II e Trici, houve baixa oscilação, com a condutividade elétrica variando entre 0,270 a $0,520 \mathrm{dS} \mathrm{m}^{-1}$, valor dentro do limite estabelecido para uso na irrigação que é de $0,700 \mathrm{dS} \mathrm{m}^{-1}$ (Ayers e Westcot, 1999). Essa baixa variação constatada nas águas superficiais se deve ao fato do poder de autodepuração dos mananciais, ou seja, a capacidade de se recuperar mesmo após receber grandes recargas (SANTI et al., 2012) (Quadro 3). Além da CE, os outros parâmetros se mantiveram abaixo do limite estabelecido. Vale ressaltar que, no semiárido brasileiro, mais especificamente na bacia do rio Acaraú, Ceará, Eloi et al. (2014) encontraram concentrações semelhantes de cloreto que variaram de 0,14 a 1,30 $\mathrm{mmol}_{\mathrm{c}} \mathrm{L}^{-1}$. 


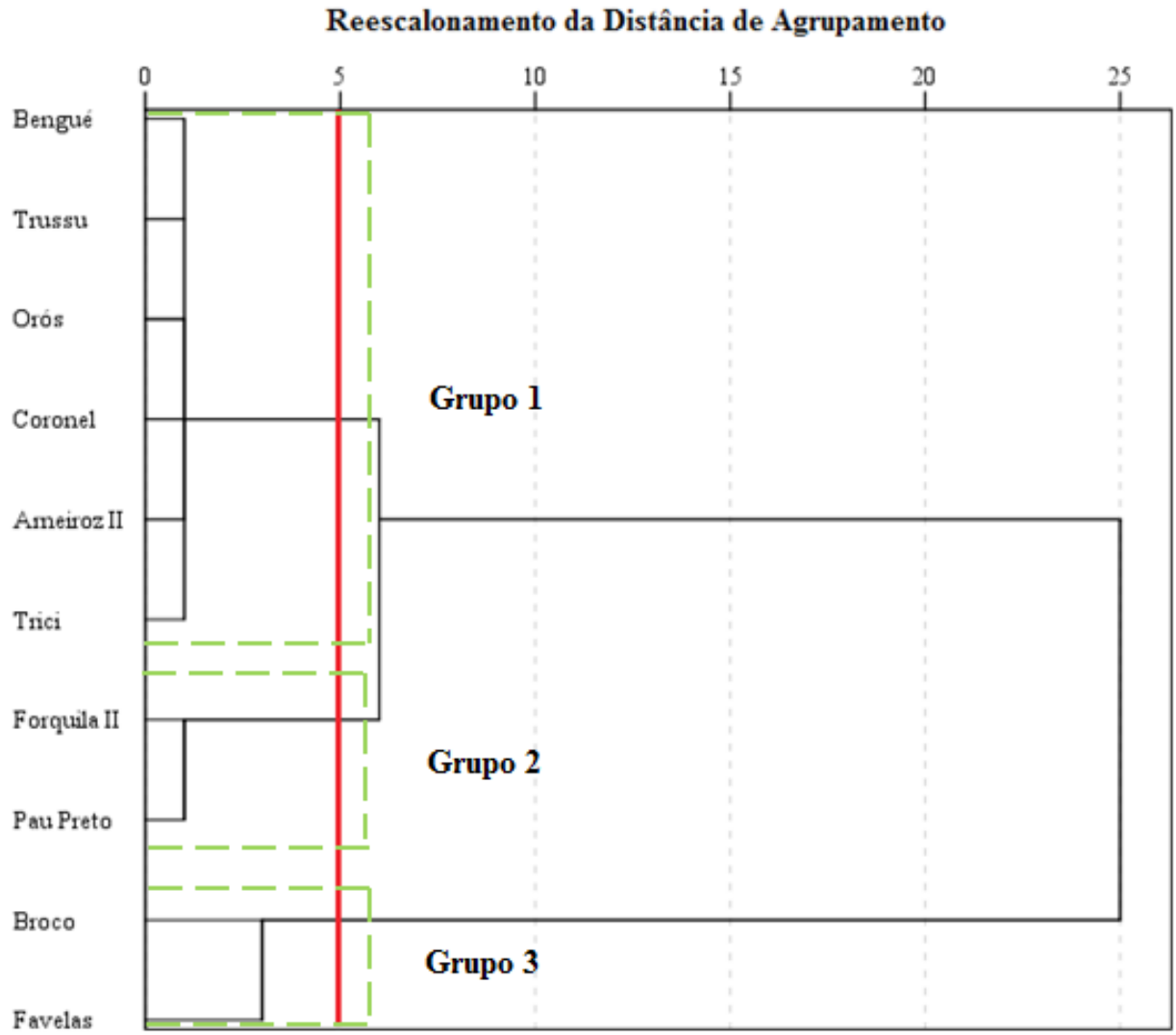

Fonte: Autor.

Figura 3. Similaridade entre os reservatórios da sub-bacia do Alto Jaguaribe, Ceará, Brasil.

Quadro 3. Valores médios das variáveis em estudo para os grupos similares definidos pela técnica da AAH para as águas superficiais da sub-bacia do Alto Jaguaribe, Ceará.

\begin{tabular}{|c|c|c|c|c|c|c|c|}
\hline \multirow{2}{*}{ Variáveis } & \multirow{2}{*}{ Estatística } & \multicolumn{6}{|c|}{ Águas superficiais da sub-bacia do Alto Jaguaribe } \\
\hline & & Grupo 1 & & Grupo 2 & & Grupo 3 & \\
\hline \multirow{3}{*}{$\mathrm{CE}\left(\mathrm{dS} \mathrm{m} \mathrm{m}^{-1}\right)$} & Média \pm DP & $\mathbf{0 , 3 4} \pm 0,09$ & $\mathrm{a}$ & $\mathbf{0 , 7 0} \pm 0,27$ & $\mathrm{a}$ & $\mathbf{1 , 8 7} \pm 0,41$ & $\mathrm{~b}$ \\
\hline & Mín & 0,27 & & 0,50 & & 1,58 & \\
\hline & Máx & 0,52 & & 0,89 & & 2,16 & \\
\hline \multirow{3}{*}{$\mathrm{Na}^{+}\left(\mathrm{mmol}_{\mathrm{c}} \cdot \mathrm{L}^{-1}\right)$} & Média \pm DP & $\mathbf{1 , 8 7} \pm 0,52$ & $\mathrm{a}$ & $\mathbf{2 , 8 2} \pm 0,01$ & $\mathrm{a}$ & $\mathbf{7 , 7 4} \pm 0,93$ & $\mathrm{~b}$ \\
\hline & Mín & 1,40 & & 2,82 & & 7,08 & \\
\hline & Máx & 2,84 & & 2,83 & & 8,40 & \\
\hline \multirow{3}{*}{$\mathrm{Cl}^{-}\left(\mathrm{mmol}_{\mathrm{c} .} \mathrm{L}^{-1}\right)$} & Média \pm DP & $\mathbf{1 , 3 3} \pm 0,37$ & $\mathrm{a}$ & $\mathbf{3 , 9 8} \pm 1,57$ & $\mathrm{ab}$ & $\mathbf{1 1 , 5 1} \pm 6,26$ & $\mathrm{~b}$ \\
\hline & Mín & 0,94 & & 2,87 & & 7,09 & \\
\hline & Máx & 1,96 & & 5,09 & & 15,94 & \\
\hline \multirow{3}{*}{$\mathrm{Ca}^{2+}\left(\mathrm{mmol}_{\mathrm{c} .} \mathrm{L}^{-1}\right)$} & Média \pm DP & $\mathbf{1 , 5 6} \pm 0,20$ & $\mathrm{a}$ & $\mathbf{2 , 3 8} \pm 0,03$ & $\mathrm{~b}$ & $\mathbf{1 , 3 2} \pm 0,03$ & $\mathrm{a}$ \\
\hline & Mín & 1,28 & & 2,36 & & 1,30 & \\
\hline & Máx & 1,89 & & 2,40 & & 1,35 & \\
\hline \multirow{3}{*}{$\mathrm{Mg}^{2+}\left(\mathrm{mg} \mathrm{L}^{-1}\right)$} & Média \pm DP & $\mathbf{1 , 0 9} \pm 0,18$ & $\mathrm{a}$ & $\mathbf{0 , 9 9} \pm 0,29$ & $\mathrm{a}$ & $\mathbf{4 , 0 6} \pm 0,93$ & $\mathrm{~b}$ \\
\hline & Mín & 0,90 & & 0,79 & & 2,40 & \\
\hline & Máx & 1,39 & & 1,20 & & 4,72 & \\
\hline
\end{tabular}

Fonte: Autor.

*Média seguida de letra minúscula diferem entre si na linha pelo teste de Tukey, em nível de 1\% de significância. 
O grupo 2, composto pelos reservatórios Forquilha II e Pau Preto, foi formado também por concentrações baixas de salinidade, porém diferiu estatisticamente ao nível de $1 \%$ de significância dos grupos 1 e 3 por causa da variável $\mathrm{Ca}^{2+}$, com valor médio de $2,38 \mathrm{mmol}_{\mathrm{c}} \mathrm{L}^{-1}$, que segundo Silva Junior e Medeiros (2007) águas com elevadas concentrações de Cálcio, quando usadas para irrigação, podem causar entupimento dos emissores ou incrustações nas tubulações de irrigação. Os íons $\mathrm{Na}^{+}$e $\mathrm{Cl}^{-}$obtiveram médias abaixo do limite estabelecido, que é de $3 \mathrm{mmol}_{\mathrm{c}} \mathrm{L}^{-1}$, assim como $\mathrm{Ca}^{2+} \mathrm{e} \mathrm{Mg}^{2+}$, com valores de 1,56 e 1,09 mmolc L $\mathrm{L}^{-1}$, respectivamente.

Os reservatórios Broco e Favelas foram os responsáveis pela formação do grupo 3, que obtiveram os maiores teores de sais dentre os reservatórios. $\mathrm{O}$ grupo 3 teve como média para a $\mathrm{CE} 1,87 \mathrm{dS} \mathrm{m}^{-1}$, valor bem superior ao limite estabelecido $\left(0,700 \mathrm{dS} \mathrm{m}^{-1}\right)$. Águas com concentrações de sais elevadas podem tornar o solo salino, impossibilitando o cultivo agrícola. Além da $\mathrm{CE}$, o $\mathrm{Na}^{+}$e $\mathrm{Cl}^{-}$estão $158 \%$ e $283 \%$, respectivamente, além do limite proposto por Ayers e Westcot (1999) para fins de irrigação. Isso impossibilita a utilização para qualquer cultura, tendo em vista a não resistência das plantas a condições tão extremas de salinidade.

A espacialização da CE na sub-bacia do Alto Jaguaribe pode ser observada na Figura 4. Os reservatórios Bengué e Favelas obtiveram os menores e maiores valores de concentrações, respectivamente. $\mathrm{O}$ reservatório Favelas detêm a maior concentração entre os açudes monitorados $\left(2,158 \mathrm{dS} \mathrm{m}^{-1}\right)$, valor bem superior ao limite estabelecido por Ayers e Westcot (1999) para irrigação que é de $0,700 \mathrm{dS} \mathrm{m}^{-1}$. Devido à reduzida capacidade volumétrica desse reservatório, há uma tendência de secar rapidamente, aumentando a concentração dos sais nas águas, além de que há uma baixa altura pluviométrica na região de Tauá, município a qual pertence o reservatório, acarretando uma não diluição dos sais. Caitano et al. (2012) estudando a avaliação de modelos e espacialização da qualidade das águas superficiais do estado do Ceará diagnosticaram o açude Favelas como sendo um dos reservatórios de maiores concentrações de sais em todo o estado do Ceará. Podemos constatar ainda uma elevada concentração nos reservatórios da região dos Inhamuns, mais precisamente próximo a cidade de Tauá, Ceará, que detêm 4 dos 10 reservatórios estudados (Broco, Favelas, Forquilhas II e Trici).

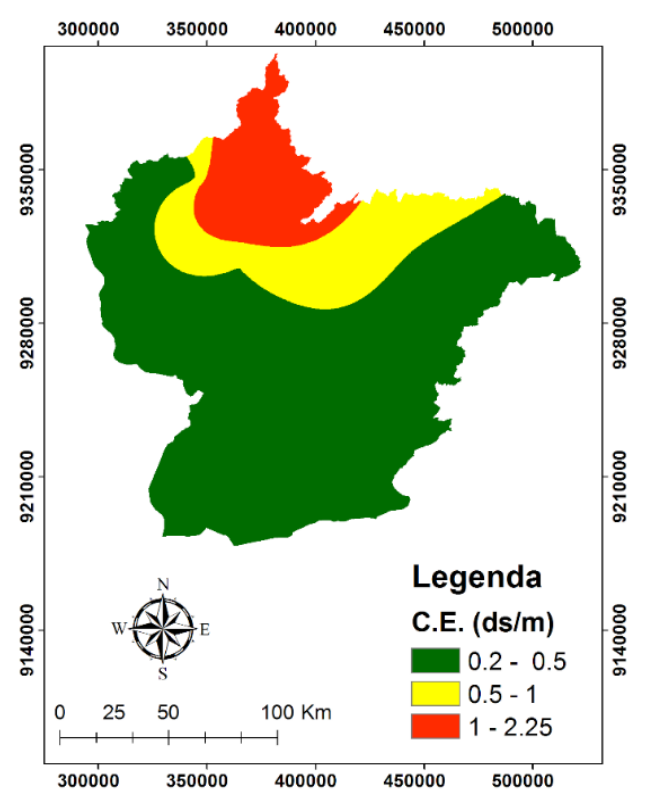

Fonte: Autor.

Figura 4. Espacialização da variável condutividade elétrica nos reservatórios da bacia do Alto Jaguaribe, Ceará, Brasil.

A espacialização para os íons $\mathrm{Cl}^{-}$e $\mathrm{Na}^{+}$ obtiveram 4 e 3 classes, respectivamente. Podese observar que os maiores valores para ambos os elementos foram encontrados na região Noroeste da bacia (Figura 5). Estas maiores concentrações estão intrínsecas aos reservatórios do município de Tauá, Ceará, que vêm sofrendo acúmulo de sais, fato comprovado pela Figura 3, em que também foi a região com maiores valores de condutividade elétrica. De acordo com Ayers e Westcot (1999), elevados teores de $\mathrm{Cl}^{-}$(Figura 4a) podem ser tóxicos para diversas culturas, e que a irrigação por aspersão pode ocasionar problemas de queima das folhas e consequentes perdas de produtividade. Já o $\mathrm{Na}^{+}$(Figura 4b), quando em baixas concentrações 

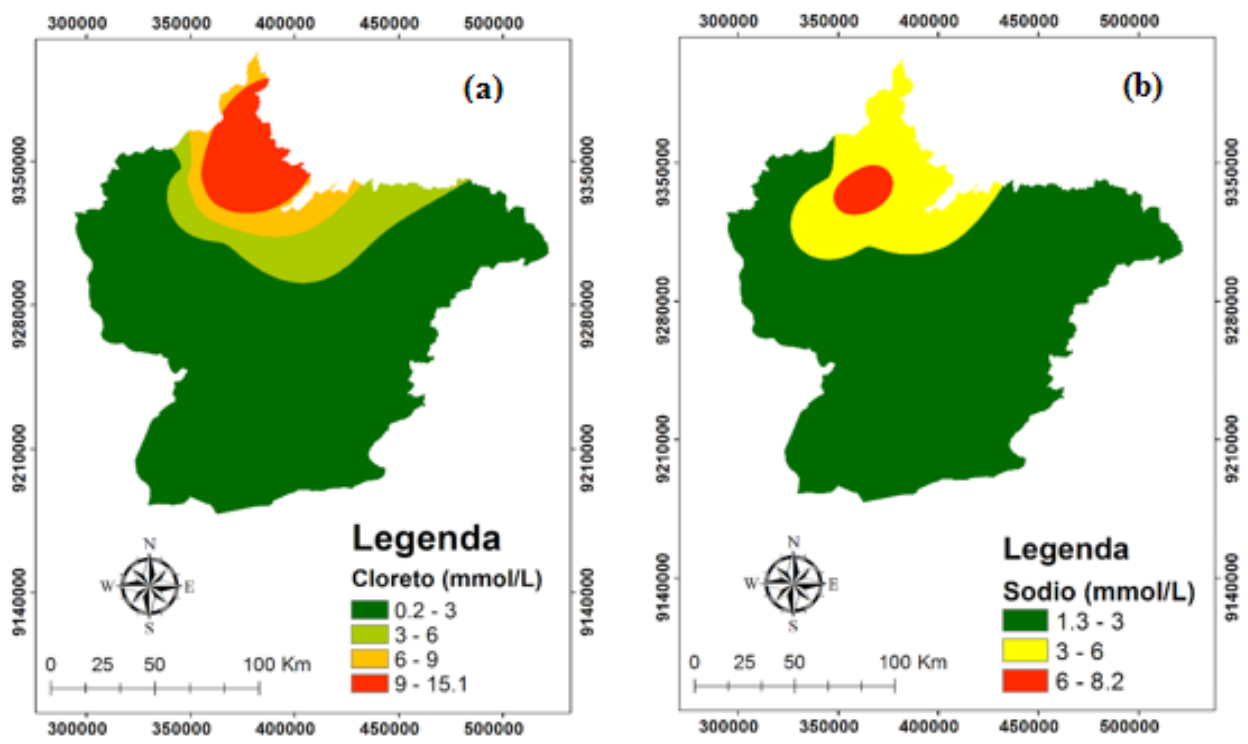

Fonte: Autor.

Figura 5. Espacialização das variáveis $\mathrm{Cl}^{-}$(a) e $\mathrm{Na}^{+}$(b) nos reservatórios da bacia do Alto Jaguaribe, Ceará, Brasil.

associados a baixas salinidades, pode provocar problemas severos de sodificação dos solos (ANDRADE et al., 2006). Limitações quanto ao íon $\mathrm{Na}^{+}$também foram encontrados por Lobato et al. (2008) avaliando a sazonalidade na qualidade da água do Distrito Irrigado Baixo Acaraú, Ceará.

A soma das concentrações de $\mathrm{Ca}^{2+}+\mathrm{Mg}^{2+}$ geraram a criação de 4 classes bem definidas na espacialização da bacia do Alto Jaguaribe (2-3 mmol $_{c} \mathrm{~L}^{-1} ; 3-4 \mathrm{mmol}_{\mathrm{c}} \mathrm{L}^{-1} ; 4-5 \mathrm{mmol}_{\mathrm{c}} \mathrm{L}^{-1} ; 5-6$ $\mathrm{mmol}_{\mathrm{c}} \mathrm{L}^{-1}$ ) (Figura 6). A maior parte da bacia se enquadrou nas classes de menores concentrações (2-3 e 3-4 $\mathrm{mmol}_{\mathrm{c}} \mathrm{L}^{-1}$ ), evidenciando que não apresentam restrições para irrigação segundo recomendações propostas por Ayers e Westcot (1999). Porém, assim como nos demais mapas a região do município de Tauá, Ceará apresentou elevadas concentrações, ultrapassando o limite estabelecido pelo autor op cit, que é de $5 \mathrm{mmol}_{\mathrm{c}} \mathrm{L}^{-1}$. Variações das concentrações dentro de uma mesma bacia também foram encontradas por Araújo Neto et al. (2014) estudando a seleção dos indicadores da salinidade das águas superficiais para a bacia Metropolitana do Ceará. Além de Barroso (2011) avaliando as águas superficiais da região Centro Sul do estado do Ceará.

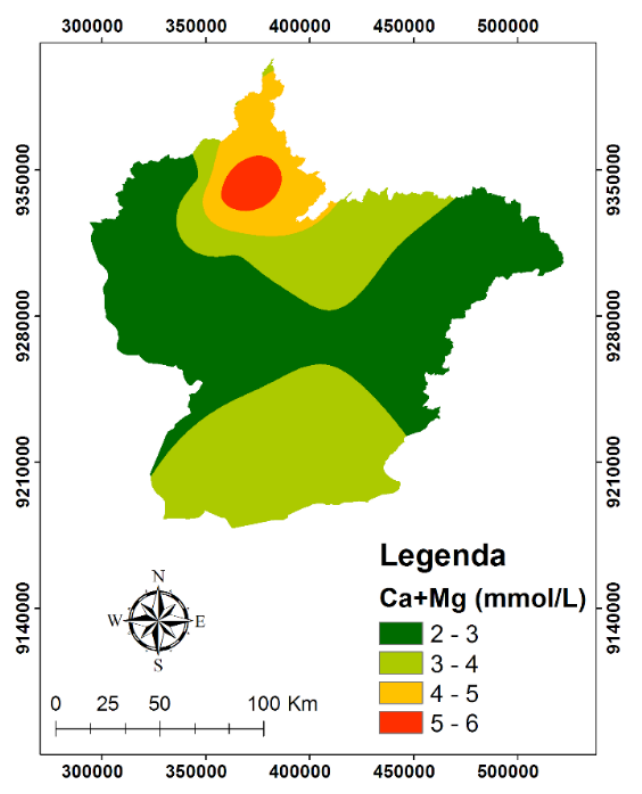

Fonte: Autor.

Figura 6. Espacialização das variáveis $\mathrm{Ca}^{2+}+$ $\mathrm{Mg}^{2+}$ no reservatórios da bacia do Alto Jaguaribe, Ceará, Brasil.

\section{CONCLUSÕES}

- A sub-bacia do Alto Jaguaribe apresentou, até o momento, moderado risco à salinidade, com exceção dos reservatórios da região do

\section{REVENG 417-426p.}


Inhamuns, em que os mesmos se encontraram com elevadas concentrações de sais.

- Os açudes Bengué e Orós apresentaram as menores concentrações de sais. Já os açudes Broco e Favelas obtiveram as maiores concentrações de sais dentre os estudados, com valores acima dos limites para irrigação.

- A estatística multivariada definiu grupos heterogêneos conforme as concentrações hidroquímicas dos reservatórios.

\section{REFERÊNCIAS BIBLIOGRÁFICAS}

ANDRADE, E.M.; BATISTA, T.M.; TEIXEIRA, A.S.; MEIRELES, M.; SOUSA, B.F.S. Mapa de vulnerabilidade da bacia do Acaraú, Ceará, à qualidade das águas de irrigação, pelo emprego do GIS. Revista Ciência Agronômica, v.37, n.03, p.279-286, 2006.

ARAÚJO NETO, J.R.; ANDRADE, E.M.; MEIRELES, A.C.M.; GUERREIRO, M.J.S. PALÁCIO, H.A.Q. Proposta de índice da salinidade das águas superficiais de reservatórios do Ceará, Brasil. Revista Agro@mbiente On-line, v.8, n.2, p.184-193, maio-agosto, 2014.

ARRAES, F.D.D.; ANDRADE, E.M.; PALÁCIO, H.A.Q.; FROTA, J.I.J.; SANTOS, J.C.N. Identificação dos íons determinantes da condutividade elétrica nas águas superficiais da bacia do Curu, Ceará. Revista Ciência Agronômica, Fortaleza, v.40, n.3, p.346-355, 2009.

AYERS, R.S.; WESTCOT, D.W. A qualidade da água na agricultura. 2.ed. Campina Grande: UFPB, 1999, p.218. (Estudos FAO: Irrigação e Drenagem, 29).

BARBOSA, M.A.L.V. Os impactos ambientais causados pela monocultura da cana-de açúcar no município de Americano do Brasil. 2006. 30f. Trabalho de Conclusão de Curso (Graduação em Geografia) - Fundação Educacional de Anicuns, Faculdade de Educação e Ciências Humanas, Anicuns, 2006.
BARROSO, A.A.F.; GOMES, G.E.; LIMA, A.E.O. PALÁCIO, H.A.Q.; LIMA, C.A. Avaliação da qualidade da água para irrigação na região Centro Sul no Estado do Ceará. Revista Brasileira de Engenharia Agrícola e Ambiental, Campina Grande, v.15, n.6, p.588-593, 2011.

BUENO, L.F.; GALBIATTI, J.A.; BORGES, M.J. Monitoramento de variáveis de qualidade de água no horto Ouro Verde-Conchal-SP. Engenharia Agrícola, Jaboticabal, v.25, n.3, p.742-748, 2005.

CAITANO, R.F.; LOPES, F.B.; CHAVES, L.C.G.; ANDRADE, E.M. Avaliação de modelos de interpolação e espacialização da qualidade das águas superficiais do Estado do Ceará. In: I Inovagri International Meeting e IV Workshop de Inovações Tecnológicas na Irrigação - Winotec, 2012, Fortaleza. Anais... Fortaleza: INOVAGRI, p.1-5, 2012.

ELOI, W.M.; SALES, M.A.L.; LIRA, J.V.; SALES, M.L.M.; NASCIMENTO, N.V.; SOUZA, J.V.R.S. Sazonalidade na qualidade da água de irrigação em açudes da bacia do rio Acaraú, Ceará. Revista Brasileira de Agricultura Irrigada, v.8, n.3, p.247-255, 201

FECHINE, J.A.L.; GALVINCIO, J.D. Agrupamento da precipitação mensal da bacia hidrográfica do rio Brigida-PE, através da multivariada. Revista Brasileira de Geografia Física, Recife, v.1, n.1, p.39-46, 2008.

FERREIRA, K.C.D.; LOPES, F.B.; ANDRADE, E.M.; MEIRELES, A.C.M.; SILVA, G.S. Adaptação do índice de qualidade de água da National Sanitation Foundation ao semiárido brasileiro. Revista Ciência Agronômica, v.46, n.2, p.277-286, 2015.

FROTA JÚNIOR, J.I.; ANDRADE, E.M.; MEIRELES, A.C.M.; BEZERRA, A.M.; SOUZA, B.F.S. Influência antrópica na adição de sais no trecho perenizado da bacia hidrográfica do Curu, Ceará. Revista Ciência Agronômica, Fortaleza, v.38, n.2, p.142-148, 2007. 
GRATTAN, S.R. Irrigation water salinity and crop production. Disponível em: <http:// anrcatalog.ucdavis.edu/pdf/8066.pdf $>$. Acesso em: 15 jul. 2005.

LOBATO, F.A.O.; ANDRADE, E.M.; MEIRELES, A.C.M.; CRISÓSTOMO, L.A. Sazonalidade na qualidade da água de irrigação do Distrito Irrigado Baixo Acaraú, Ceará. Revista Ciência Agronômica, v.39, n.1, p.167-172, 2008.

LOPES, F.B. ANDRADE, E.M.; AQUINO, D.N.; LOBATO, F.A.O.; MENDONÇA, M.A.B. Indicadores de sustentabilidade do Perímetro Irrigado Baixo Acaraú, Ceará, empregando a análise multivariada. Revista Ciência Agronômica, Fortaleza, v.40, n.1, p.17-26, 2009.

MENDES, J.S.; CHAVES, L.H.G.; CHAVES, I. B. Qualidade de águas para fins de irrigação da região do Congo, PB. Revista Caatinga, Mossoró, v.21, n.2, p.131-138, 2008.

PALÁCIO, H.A.Q.; ARAÚJO NETO, J.R.; MEIRELES, A.C.M.; CHAVES, L.C.G. Similaridade e fatores determinantes na salinidade das águas superficiais do Ceará, por técnicas multivariadas. Revista Brasileira de Engenharia Agrícola e Ambiental, Campina Grande, v.15, n.4, p.395-402, 2011.

PALÁCIO, H.A.Q. ANDRADE, E.M.; LOPES, F.B.; D’ALMEIDA, D.M.B.A.; ARRAES, F.D.D. Similaridade da qualidade das águas superficiais da bacia do Curu, Ceará. Ciência Rural, Santa Maria, v.39, n.9, p.2494-2500, 2009.

SALES, M.M.; ARAÚJO NETO, J.R.; PALÁCIO, H.A.Q.; CHAVES, L.C.G.; GOMES, F.E.F.
Variação espacial e modelagem da salinidade das águas superficiais de reservatórios da bacia metropolitana do Ceará. Revista Brasileira de Agricultura Irrigada, v.8, n.5, p.354-365, 2014.

SALGADO, E.V.; ANDRADE, E.M.; FONTENELE, S.B.; MEIRELES, A.C.M. Similaridade das variáveis hidroquímicas com o uso da análise multivariada, na bacia do Salgado, Ceará. Revista Caatinga, Mossoró, v.24, n.3, p.158-166, jul.-set., 2011.

SANTI, G.M.; FURTADO, C.M.; MENEZES, R.S.; KEPPELER, E.C. Variabilidade espacial de parâmetros e indicadores de qualidade da água na sub-bacia hidrográfica do igarapé São Francisco, Rio Branco, Acre, Brasil. Ecologia Aplicada, v.11, n.1, p.23-31, 2012.

SANTOS, J.C.N.; MEIRELES, A.C.M.; ANDRADE, E.M.; ARAUJO NETO, J.R. Modelagem da concentração de sódio, cálcio e magnésio nas águas superficiais da bacia do rio Acaraú. In: XIX Congresso Nacional de Irrigação e Drenagem - CONIRD, Montes Claro. Anais... Montes Claros - MG, 2009.

SILVA JÚNIOR, J.N; SOUSA, A.R.; SÁ, V.A.L.; LIMA, B.P. Relações entre a concentração de íons e a salinidade de águas subterrâneas e superficiais visando à irrigação no sertão de Pernambuco. Revista Brasileira de Engenharia Agrícola e Ambiental, Campina Grande, v.4, n.2, p.189-193, 2000 .

SILVA JÚNIOR, M.J; MEDEIROS, J.F. Desenvolvimento de obstruções em gotejadores e eficiência do controle usando dois tipos de ácidos. Revista Irriga, v.12, n.02, p.185-191, 2007. 